\title{
Manifestation of the long-term solar cyclicity in climate archives over 10 millennia
}

\author{
V.A.Dergachev \\ Ioffe Physico-Technical Institute, Russian Academy of Sciences, Politekhnicheskaya str. 26, \\ 194021 St.Petersburg, Russia e-mail: v.dergachev@pop.ioffe.rssi.ru
}

\begin{abstract}
Many records of climatic and environmental changes based on various proxy data exhibit distinct cyclicities that have been attributed to extraterrestrial forcing such as changes in solar activity. To study the long-term changes of solar activity, different data sets of solar activity, both indirect data (the Schove series) and proxy data (cosmogenic isotopes) were analyzed. Both sets of solar activity clearly show centennial and millennial changes.
\end{abstract}

\section{Introduction}

From the latter part of the 19th century, there were many claims to found connections between sunspots and climate. The variable nature of the Sun has almost always been accompanied by efforts to find possible impacts on the Earth through comparisons with weather and climate records. After more than a century of controversy, the debates concerning the solar variability effect on climate of the Earth remains to be settled.

We know that there are significant changes of much longer term in the overall level of solar activity. Sunspots have been observed and recorded by astronomers since the early 1600 s, and long-term changes in their number are clearly evident. A point that should be mentioned is that three episodes of sunspot minima are now recognized during the past millennium: 1645-1715 - Maunder Minimum; 1416-1534 - Sporer Minimum; 1280-1350 Wolf Minimum (Nesje \& Dahl, 2000) - changes of much longer term in the overall level of solar activity. Long-term changes in sunspots number are clearly evident. These three episodes are separated by $\sim 200$-year spans.

\section{The long-term cyclicity from the Schove's sunspot indirect data}

It is almost 50 years since Schove's indirect data set, based mainly on auroral record, which Schove transferred to approximative sunspot numbers and sunspot phase information (Schove, 1955). The series provides estimates for the years of minima and maxima and for the amplitudes of the 11-year cycles since $642 \mathrm{BC}$ to AD 1990. However, the quality of Schove's sunspot indirect data has been questioned and for that reason has not been used by most scientists in their analytical work.

However, recently it was carried out the estimation of the quality of this data set, based on the modern methods of signal processing (Jelbring, 1995). He analyzed the changes of the lengths and phases of the solar cycles. The sunspot cycle phase is the integrated deviation (in years) from an assumed constant sunspot cycle length. The check of the quality had been made by two separate methods. One looks for similar spectral patterns, analyzing parts of the whole time series and it was considered the compatibility of the spectral pictures obtained. The other method is designed to check how sensitive these spectral patterns are when adding simulated noise to the basic proxy data set and it was checked the stability of the spectral structure, chosen in not noised data set. In this work 


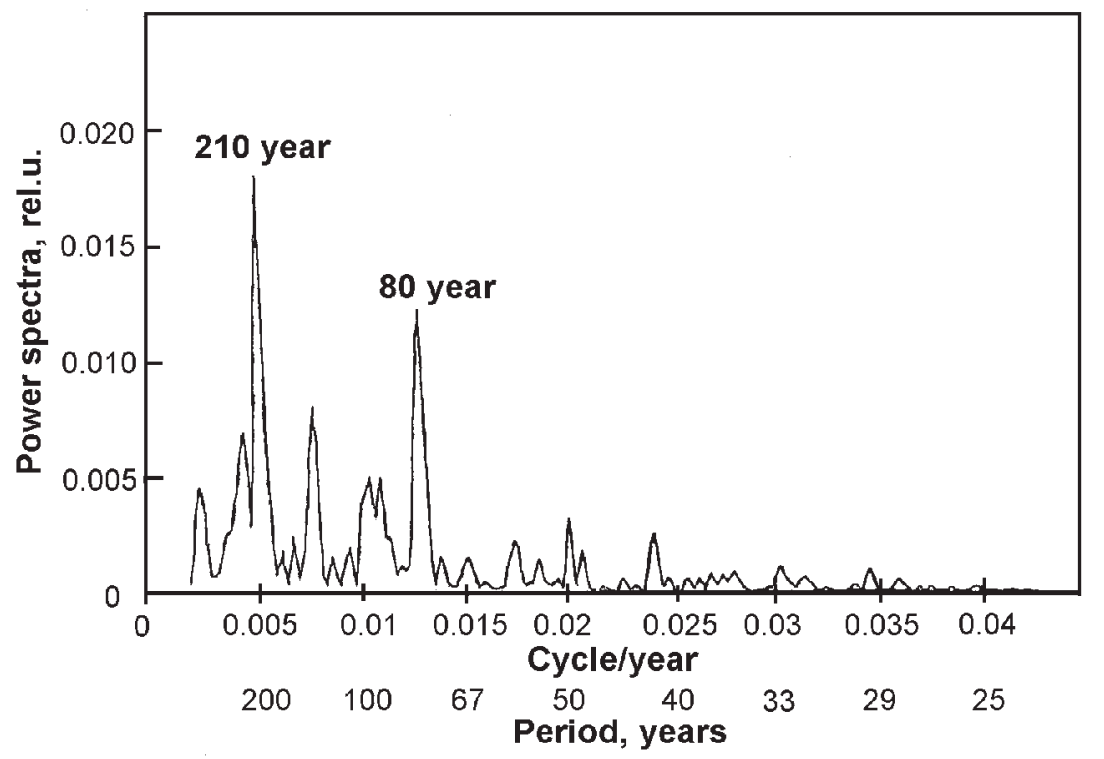

Figure 1. Power spectrum of sunspot cycle phase (magnified part of power spectrum).

it was shown that the analyzed data set to be of a high quality concerning length and phase information of the solar cycle and it may be a rather good index spreading the direct observations of solar activity at least 2000 years back in time. It was extracted the range of structured phase cycles for which the amplitudes seen very time dependent. In the spectrum one may find the dominant slowly varying in time component with a period around 1700 years. After extracting from the time series of the long-term trend in the spectrum one may detect most powerful $\sim 210$-year and $\sim 80$-year cycles (figure 1 ). It should be stress that the same harmonics are seen in solar cycle lengths of 11-year solar cycles.

Another approach was used by Komitov \& Bonev (2001) for this data set. They examined the conditions for violations of the Gnevyshev-Ohl rule, which states that the evennumbered 11-year solar cycles have been followed by higher in amplitude odd-numbered ones. The reliability of these data has been discussed. A total of 152 cycles were investigated, the last one being cycle 21 in the International Sunspot Number series. It was shown that there is a well-outlined group of even-numbered 11-year cycles that are very likely to be followed by odd-numbered ones. They used the continuous part of data, which is after AD 296. For comparison, the best reconstruction of solar activity, based on optical records, cover the period after 1610 (Hoyt \& Schatten, 1998). The authors established the association of Gnevyshev-Ohl rule violation with the long-term behavior of solar activity combining the smoothed Schove data with amplitude of the strongest evennumbered solar cycles violating the Gnevyshev-Ohl rule and connected this association with $\sim 200$ - and 2400-year cycles.

Recently, Komitov \& Kaftan (2003) performed a mathematical statistic analysis of these data using two independent methods and revealed statistically significant longterm oscillations, including 205-year period.

Thus, these investigations allow to conclude that Schove's indirect data set is found to be of a high quality concerning sunspot cycle length and phase information and may be 


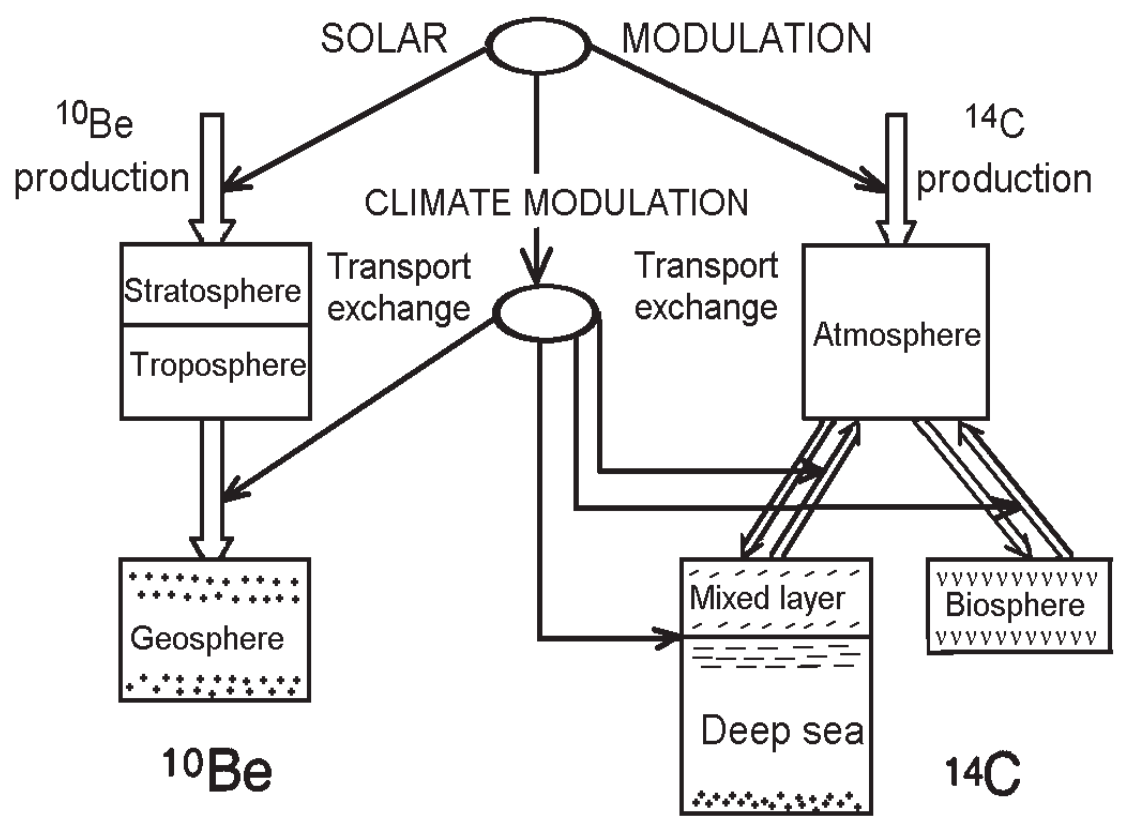

Figure 2. Solar and climatic modulation of cosmogenic isotope $\left({ }^{10} \mathrm{Be}\right.$ and $\left.{ }^{14} \mathrm{C}\right)$ production.

used as a good equivalent of solar activity changes at least 2200 years back in time. We can make a conclusion that indirect solar data sets demonstrate the long-term variations of solar activity.

\section{The long-term solar cyclicity from proxy data}

To study the long-term changes of solar activity on more extended time scales, we can use only proxy data. Much longer records of solar behavior come from some natural archives, which contain the information about the variability of solar activity in the past. Tree rings and ice cores represent natural archives that contain such information about climate change and solar variability. These archives include cosmogenic isotopes that are produced in the Earth's atmosphere by the impact of galactic cosmic rays. When the Sun is more active, its own magnetic fields deflect some of the cosmic rays that would otherwise reach the Earth. When it is less active, we receive more of them. Both climate history and the forcing factors of climatic variability can be derived from a variety of terrestrial and marine archives.

There are two the most-used cosmogenic isotopes in well-dated samples for these purposes: radiocarbon in tree-rings and berillium-10 in ice cores. Both isotopes are produced in a similar manner, when the cosmic ray flux enters the Earth's atmosphere. The physics of the production processes of these isotopes is well understood. Solar modulation affects both isotopes in a very similar manner, but climate modulation is expected to be different due to the different geochemical systems for these isotopes (figure 2). From comparison of their records one can distinguish between the solar and climatic modulation of the production origin of both isotopes. Periods of maximum ${ }^{14} \mathrm{C}$ and ${ }^{10} \mathrm{Be}$ concentration 


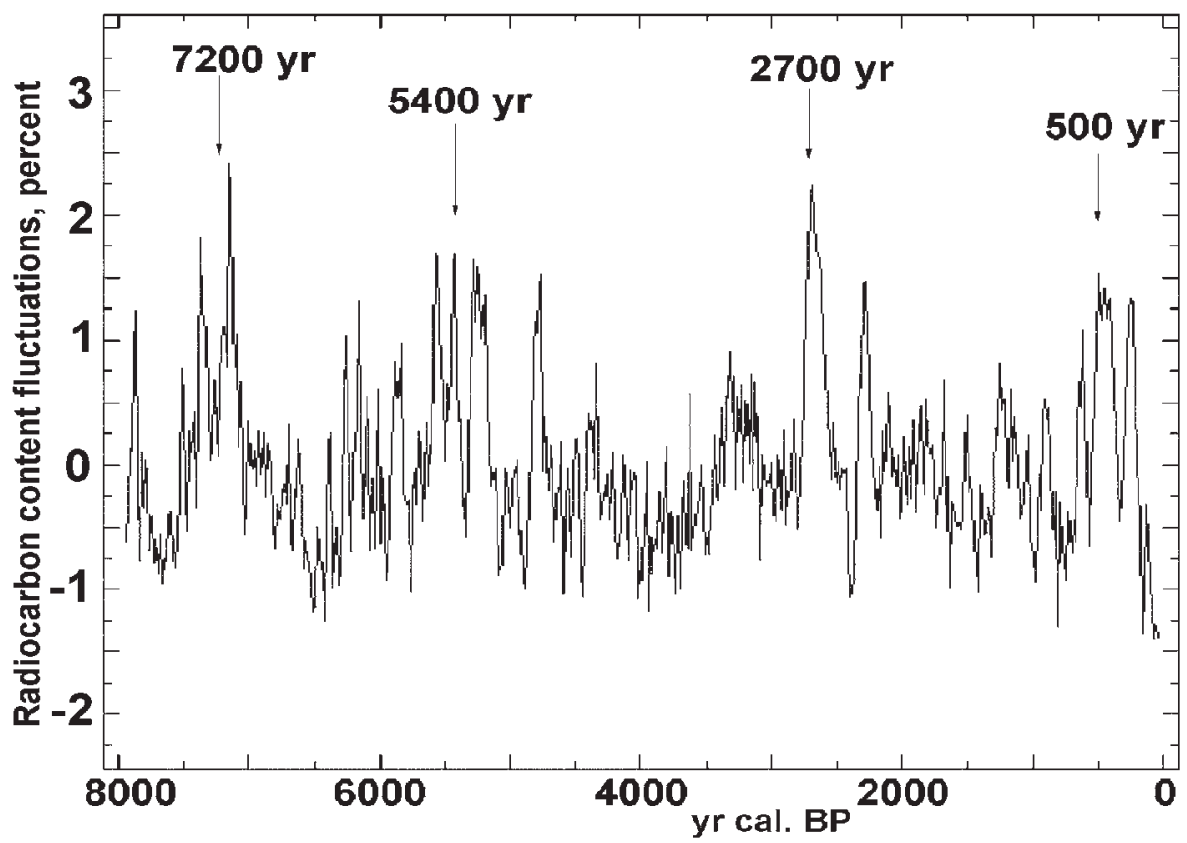

Figure 3. Reconstruction of the radiocarbon concentration (after subtraction of the long-term trend) derived from tree-rings. The arrows show the vicinity of high amplitudes of ${ }^{14} \mathrm{C}$ concentration variations.

corresponding to known solar activity minima (Maunder, Sporer, Wolf) were identified for the last millennia (see for example Bard et al. 1997; Wagneret al. 2001).

Forcing factors of climate variability can be indicated from ${ }^{14} \mathrm{C}$ concentrations in treerings (Stuiver \& Becker, 1993), over the past 8000 years (figure 3). Upon subtraction from the initial radiocarbon time series of the long-term trend, the residual time series clearly shows centennial and millennial changes. The major oscillations are in the vicinity of 7200, 5400, 2700 and 500 cal year BP, with $\sim 2400$-year periodicity between them. This reconstruction shows that ${ }^{14} C$ production was higher during the Little Ice Age, when solar activity was extremely low. Large-scale cyclic oscillations of the ${ }^{14} C$ concentration with $\sim 2400$-year cycle have been suggested to correspond to phases of cold climate (Dergachev \& Chistyakov, 1995).

Investigations of mountain glaciers during the Little Ice Age $(\sim 500$ years ago $)$ show a few periods of glacial growth associated with several low temperature oscillations, and some of them correspond to high amplitude oscillations in the ${ }^{14} C$ concentration record.

Mathematical methods of spectral analysis can give important information on the presence of a cyclicity in time series of natural processes. Spectral analysis of the available long-term series of the ${ }^{14} C$ concentration of tree-rings, using the classical harmonic methods of time series analysis, showed spectral lines at a number of periods. However, the same harmonic features are not independent. The dynamics of variations in the ${ }^{14} C$ concentration in dated samples has been studied through the utilization of various methods of Fourier spectral analysis by Sonett (1984), and by Damon \& Sonnet (1991). The first 


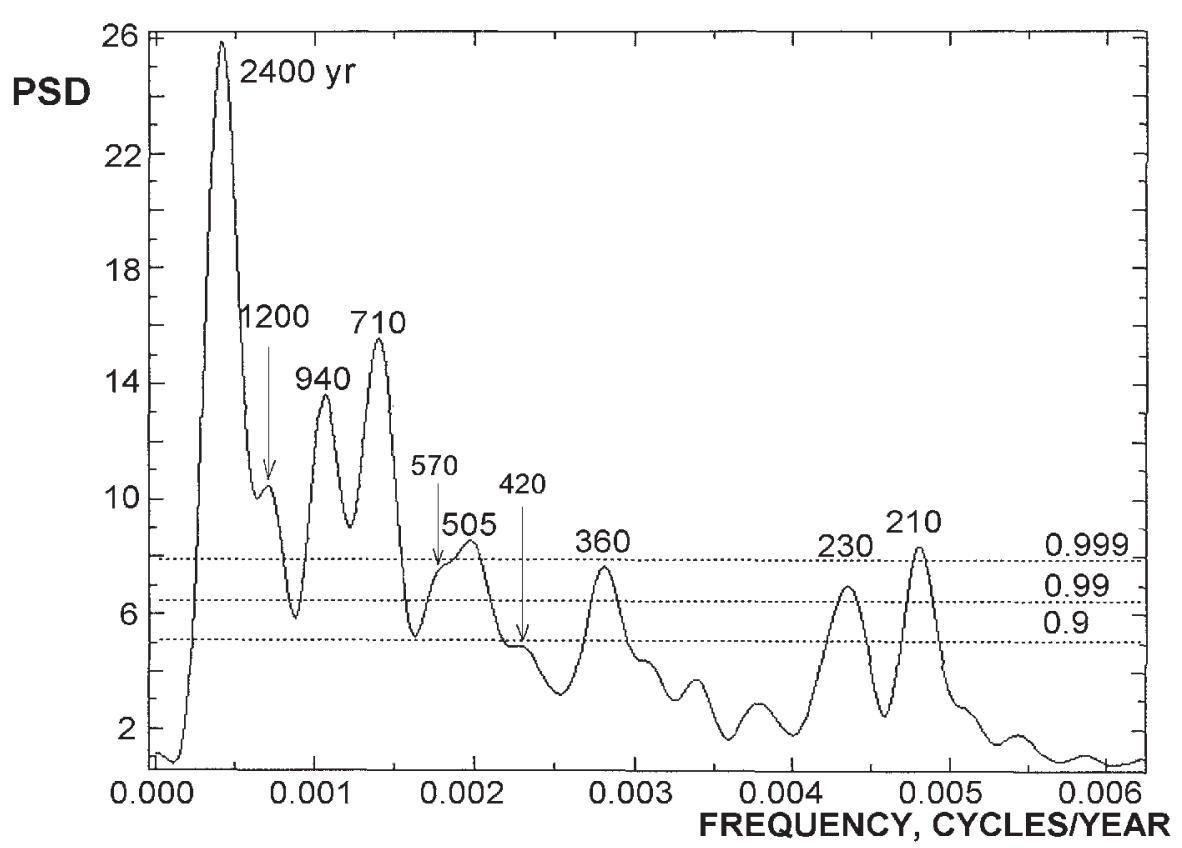

Figure 4. The normalized power spectrum of ${ }^{14} C$ time series for the last 8000 years obtained by the Blackman-Tukey method after removal of the long-term trend in the raw data series. The dotted lines are the confidence levels. The numbers near the peaks are periods in years.

detection of 2200-2400-year quasi-periodicity in the long ${ }^{14} C$ concentration record from tree rings was made by Suess (1980).

Let us consider the results of some recent spectral analyses of variability in long-term data. Vasiliev \& Dergachev (2002) conducted a spectral analysis of the non-stationary behavior of high-precision ${ }^{14} C$ concentration data observed in tree-rings (Stuiver \& Becker, 1993). Several methods of spectral analysis were utilized: power spectrum, time-spectrum, band-pass filtering and bi-spectrum. It was demonstrated that the amplitude of largescale fluctuations varied periodically. Period of this change is approximately 2400 years. The bi-spectrum analysis of data demonstrates the existence of amplitude modulation. The power spectrum in figure 4 shows a very strong line with the period of ca. 2400 years, with an amplitude significantly exceeding the amplitudes of other lines in the spectrum, i.e. the component with period of $\sim 2400$ years is the principle component of long-term changes in the radiocarbon time series.

Thus, both indirect indicators of solar activity (auroral record, naked-eye sunspots in the past) and well-dated records of proxy data (radiocarbon and berillium-10) exhibit the long-time cyclic variations of about 2400, 200 and 80 years, as well as more shorter.

\section{Acknowledgements}

This work is supported by RFBR, project No. 03-04-48769 and INTAS, project No. 2001-0550 and No. 03-51-4445. 


\section{References}

Bard, E., Raisbeck, G., Yiou, F. \& Jouzel, J. 1997 Earth and Plane. Sci. Let. 150, 453-462.

Damon, P.E. \& Sonett, C.P. 1991 In The Sun in Time (ed. C.P.Sonett, M.S.Giampapa, \& M.S.Matthews). 360-388. Univ. of Arizona Press, Tucson.

Dergachev, V.A. \& Chistyakov, V.F. 1995 Radiocarbon 37, 417-424.

Hoyt, D.V. \& Schatten K.H. 1998 Solar Phys. 181, 491- 512.

Jelbring, H. 1995 J. of Coastal. Research, Special Issue 17, 363-369.

Komitov, B. \& Bonev, B. 2001 Astrophys. J. Lett. 554, L119-L122.

Komitov, B. \& Kaftan, V.I. 2003 Geomagnetism and Aeronomy 43, 553-561.

Nesje, A. \& Dahl, S.O. 2000 Glaciers and environmental change. Arnold, London, 203 p.

Schove, D.J. 1955 J. Geophys. Res. 60, 127-146.

Sonett, C.P. 1984 Rev. of Geophys. \&3 Space Phys. 22, 239-254.

Stuiver, M. \& Becker, B. 1993 Radiocarbon 35, 35-65.

Suess, H.E. 1980 Radiocarbon, 22, 200-209.

Vasiliev, S.S. \& Dergachev, V.A. 2002 Annales Geophysicae 20, 115-120.

Wagner, G., Beer, J., Masarik, J., Muscheler, R., Kubik, P.W., Mende, W., Laj, C., Raisbeck, G.M., \& Yiou, F. 2001 Geophys. Res. Lett. 28, 303(2000GL006116). 